\title{
Polyol Pathway Exacerbated Ischemia/Reperfusion-Induced Injury in Steatotic Liver
}

\author{
Changhe Zhang, Changjun Huang, Yuan Tian, and Xiangcheng Li \\ Department of Liver Transplantation, The First Affiliated Hospital of Nanjing Medical University, Nanjing, Jiangsu 210029, China \\ Correspondence should be addressed to Xiangcheng Li; doclixiangcheng@sohu.com
}

Received 20 January 2014; Revised 14 April 2014; Accepted 16 April 2014; Published 21 May 2014

Academic Editor: Felipe Dal-Pizzol

Copyright (c) 2014 Changhe Zhang et al. This is an open access article distributed under the Creative Commons Attribution License, which permits unrestricted use, distribution, and reproduction in any medium, provided the original work is properly cited.

\begin{abstract}
Background. The polyol pathway, a bypass pathway of glucose metabolism initiated by aldose reductase (AR), has been shown to play an important role in mediating tissue ischemia/reperfusion (I/R) impairment recently. Here, we investigated how and why this pathway might affect the fatty liver following I/R. Methods. Two opposite models were created: mice with high-fat-diet-induced liver steatosis were treated with aldose reductase inhibition (ARI) and subsequent I/R; and AR-overexpressing L02 hepatocytes were sequentially subjected to steatosis and hypoxia/reoxygenation. We next investigated (a) the hepatic injuries, including liver function, histology, and hepatocytes apoptosis/necrosis; (b) the $\mathrm{NAD}(\mathrm{P})(\mathrm{H})$ contents, redox status, and mitochondrial function; and (c) the flux through the caspase-dependent apoptosis pathway. Results. AR-inhibition in vivo markedly attenuated the I/Rinduced liver injuries, maintained the homeostasis of $\mathrm{NAD}(\mathrm{P})(\mathrm{H})$ contents and redox status, and suppressed the caspase-dependent apoptosis pathway. Correspondingly, AR overexpression in vitro presented the opposite effects. Conclusion. The flux through the polyol pathway may render steatotic liver greater vulnerability to I/R. Interventions targeting this pathway might provide a novel adjunctive approach to protect fatty liver from ischemia.
\end{abstract}

\section{Introduction}

Despite the last hope for selective patients with end-stage liver disease, liver transplantation is facing the dilemma of a huge donor deficit. Today, this worry has been further compounded by the increasing proportion of fatty liver grafts [1]. As fatty livers increase the likelihood of developing postoperation primary nonfunction compared with lean ones, macrovesicular steatosis within more than $30 \%$ of hepatocytes is a widely recognized exclusion criterion for candidate liver donors $[1,2]$. On the other hand, patients with liver steatosis also have a greater risk of developing liver dysfunction after major hepatectomy, in which portal triad occlusion is routinely utilized to reduce blood loss. The causes of the increased vulnerability of fatty livers in such circumstances have not been fully clarified but may be predominantly associated with excessive oxidative-stressbased injury [1].

The polyol pathway, which consisted of the two key enzymes aldose reductase (AR) and sorbitol dehydrogenase
(SDH), has been implicated in the pathogenesis of various diabetic complications. In this bypass pathway, glucose is reduced to sorbitol by AR with the concomitant oxidation of coenzyme NADPH to NADP, and then SDH converts sorbitol to fructose with the reduction of NAD to NADH. Recently, accumulating evidences have indicated the detrimental role of polyol pathway in the I/R events, which could be reversed by ARI [3-5]. Till now, the potential mechanisms underlying this beneficial effect have not been fully elucidated. Meanwhile, liver is rarely involved as the target organ. Therefore, this study was designed to explore how and why the ARI might affect the steatotic liver subjected to ischemia/reperfusion (I/R) insult.

\section{Material and Methods}

The study was approved by the Nanjing Medical University Experimental Animal Department (number NJMUAEARIA-4001-20120401) and complied closely with the Guide for the Care and Use of Laboratory Animals published 
by the US National Institutes of Health (NIH publication number 85-23, revised 1996).

2.1. Reagents. The AR inhibitor (zopolrestat), oleate, and palmitate were all purchased from Sigma (St. Louis, MO, USA). The triglyceride determination kits were from Sigma Chemical Company. NADP(H), NAD(H), and Annexin VFITC/PI Quantification Kits were from Biovision. Diagnostic kits for JC-1, malondialdehyde (MDA), glutathione (GSH)/oxidized glutathione (GSSG), reactive oxygen species (ROS), and BCA protein assay kits were all from the Beyotime Institute of Biotechnology (Nanjing, China). Primary antibodies from rabbit directed against Bcl-2, Bax, cleaved caspase 3 , and $\beta$-actin were all purchased from Cell Signaling Technology (Cat. \# 3498, 2772, 9664, and 5125, sequentially). The primary antibody from rabbit directed against AR and the secondary antibody from mouse were from Abcam (Cat. \# 175394 and 99697, resp.).

2.2. Models of Fatty Liver and I/R. Wild-type C57BL/6 male mice (aged 2 weeks and weighted 9-10 g) were obtained from the Chinese Academy of Sciences (SLRC, Shanghai, China), maintained in $25 \pm 2^{\circ} \mathrm{C}$ under a $12 \mathrm{~h}$ dark/light cycle, and fed high-fat diet (HFD: 20\% lard, 4\% sucrose, $2 \%$ milk, $1 \%$ cholesterol, and $73 \%$ standard chow) to develop fatty livers. Six weeks later, the mice were randomized into three groups containing 11 animals each: AR inhibition (ARI) group, pretreated with intraperitoneal administration of zopol (50 mg/kg/day) for 5 days + 70\% liver I/R; control (Ctrl) group, treated with saline control $+70 \%$ liver I/R; and Sham group, administrated with intraperitoneal administration of zopol $(50 \mathrm{mg} / \mathrm{kg} / \mathrm{day})$ for 5 days + sham-operation. Before the intraperitoneal injection, one random animal from each group was sacrificed and hepatic sections were stained with Oil Red $\mathrm{O}$ to validate the steatosis as previously described [6]. Twenty-four hours after the last drug treatment, the mice were subjected to $1 \mathrm{~h}$ of $70 \%$ hepatic ischemia and subsequent 6 or $24 \mathrm{~h}$ of reperfusion, as described previously [7]. Serum samples were collected to measure alanine aminotransferase (ALT) and aspartate aminotransferase (AST). The liver lobes subjected to I/R were harvested. The animals were ultimately killed under deep anesthesia.

2.3. Steatosis Liver Triglyceride Determination. Frozen liver tissues were homogenized and the crude lipids were extracted with the chloroform : methanol $(2: 1)$. After the removal and blow-drying of chloroform phase, the samples were resuspended in chloroform-Triton $\mathrm{X}$, redried under nitrogen, and eventually resuspended in distilled water. The concentration of triglyceride (TG) was measured in a 96-well plate using a colorimetric assay kit.

2.4. Detections of the ROS Generation and Apoptosis/Necrosis of Fatty Liver by Flow Cytometry (FCM). Single-hepatocyte suspensions were prepared using a modified enzymatic technique as previously described, and subsequent Trypan Blue exclusion demonstrated that cell viability exceeded $89 \%$ for all animals [8]. The percentage of ROS-positive hepatocytes was then determined by a FACScan cytometer (Becton Dickinson) using the redox-sensitive dye DCFH-DA, according to the manufacturer's instruction. The degrees of hepatocyte apoptosis and necrosis were also quantified with FCM using the Annexin V-FITC Kit and the Flow J software. The upper right, upper left, and lower right quadrants represented the late apoptotic, necrotic, and early apoptotic cells, respectively. The total proportion of apoptotic cells was then calculated by adding the proportions of late and early apoptosis.

2.5. Biochemical Measurements in Fatty Liver. The cytosolic contents of $\mathrm{NAD}(\mathrm{P})(\mathrm{H})$ and ratios of NAD/NADH and $\mathrm{NADP} / \mathrm{NADPH}$ were determined using quantification kits. Fresh liver samples were homogenized in homologous extraction buffer and centrifuged at $14,000 \mathrm{rpm}$ for $5 \mathrm{~min}$. The supernatants were filtered through $10 \mathrm{kD}$ Spin Columns (BioVision, Cat. \# 1997-25) to cut off the NAD(P)H-consuming enzyme NADase and then measured according to the manufacturer's instructions.

The concentrations of total glutathione (GSH) and its oxidized disulfide (GSSG) were detected using a secondstep enzymatic method, as previously described [9]. The concentration of reduced GSH was obtained by subtracting the GSSG concentration from the total GSH concentration.

To measure MDA, frozen liver samples were homogenized in $20 \mathrm{mM}$ trisbuffer (pH 7.4) containing $5 \mathrm{mM}$ butylated hydroxytoluene and centrifuged at $4000 \times \mathrm{g}$ for $15 \mathrm{~min}$ as previously described [10]. All further steps were performed under the guidance of the protocol contained in the commercial kits (Beyotime, Cat. \# S0131).

2.6. Morphological Observations. Histological sections stained with hematoxylin and eosin (H\&E) were processed routinely. The degrees of necrosis and inflammation in the mouse livers were evaluated by one experienced pathologist using the Suzuki score, which measures the extent of congestion, vacuolization, and necrosis on a four-point scale for a total score of $0-12$ [11]. For ultrastructural observations, ultrathin $(70 \mathrm{~nm})$ slices of the ischemic livers were conventionally prepared for scanning electron microscopy (SEM) and interpreted by a pathologist blinded to the groups.

2.7. Model of AR Overexpression, Steatosis, and Hypoxia/Reoxygenation $(H / R)$. To construct a hepatocellular line stably overexpressing AR, human L02 hepatocytes (Chinese Academy of Sciences, Shanghai, China) were transfected with pcDNA3.1B-AR-Flag-GFP (pAR; Chinese National Human Genome Center, Shanghai, China) using Lipofectamine 2000 (Invitrogen). Before transfection, the following primers were used to amplify the open reading frame of the AR gene: ARnest-out: $5^{\prime}$-ATTTAAAGGTACGCGCCGCG-3' (forward) and $5^{\prime}$-CGCTGGCCACTCTACAGGTT- $3^{\prime}$ (reverse); ARnest-in: $5^{\prime}$-ATATCTCGAGATGGCAAGCCGTCTCCTGCT-3' (forward) and $5^{\prime}$-GCGCGAATTCTAAACTCTTCATGGAAGGGGTAATCC- $3^{\prime}$.

To induce the steatosis in L02 hepatocyte, a modified method from previous literatures was deployed [12]. In brief, transfected with pAR (pAR group) or empty plasmid (GFP group), or without transfection (Ctrl group), hepatocytes 
were incubated in Dulbecco's modified Eagle's medium containing free fatty acids (FFA) at a final concentration of $0.6 \mathrm{mM}$ (2:1 ratio of oleate: palmitate) for $8 \mathrm{~h}$ to induce fat overloading. Hepatocytes' steatosis was assessed through measuring hepatocellular TG contents using the aforementioned method. Hepatocytes underwent pAR-transfection and no steatosis-induction was used as negative control (Lean group).

The H/R procedure was performed using an AnaeroPack$\mathrm{CO}_{2}$ (Mitsubishi Gas Chemical Co., Tokyo, Japan), as previously described [13]. Firstly, hepatocytes cultured on Petri dish were exposed to phosphate-buffered saline (nutrient deprivation). The dishes were then placed into anaerobic chamber in $37^{\circ} \mathrm{C}$ environment together with AnaeroPack$\mathrm{CO}_{2}$ which could absorb oxygen and produce carbon dioxide both to an ultimate concentration of $10 \%$ within $1 \mathrm{~h}$ (oxygen deprivation). Four hours later, the hepatocytes were reexposed to normoxic conditions $\left(95 \% \mathrm{O}_{2}\right.$ and $\left.5 \% \mathrm{CO}_{2}\right)$ and total culture medium for a further $6 \mathrm{~h}$.

2.8. Investigating L02 Hepatocytes' Apoptosis by FCM Using TUNEL-Staining. To evaluate the hepatocytes' apoptosis after H/R exposure, commercial Apo-BrdU-Red In Situ DNA Fragmentation Assay Kit (BioVision, Cat. \# K404-60) was deployed. The Br-dUTP in this kit could actively bind DNA strand breaks, then be identified by a red fluorescence labeled anti-BrdU monoclonal antibody, and ultimately be read by FCM (Ex/Em: 488/576 nm). In brief, L02 hepatocytes after $\mathrm{H} / \mathrm{R}$ treatment were collected, resuspended into PBS containing $1 \%(\mathrm{w} / \mathrm{v})$ formaldehyde, and stored in $4^{\circ} \mathrm{C}$. Further procedures were performed according to the instructions contained in the kit.

2.9. Measuring the L02 Hepatocytes' MDA Level. The apoptosis in L02 hepatocytes was explored using the aforementioned methods when L02 cell underwent H/R treatment.

2.10. Mitochondrial Depolarization Assessment. JC-1 probe is a bioimaging dye which dominantly accumulates in the mitochondria as aggregates and emits red fluorescence $(580 \mathrm{~nm})$ when mitochondrial membrane potential $(\Delta \Psi \mathrm{m})$ is high. On the contrary, JC-1 flees the mitochondria as a monomer and emits green fluorescence $(520 \mathrm{~nm})$ when $\Delta \Psi \mathrm{m}$ is low. Here, the mitochondrial depolarization in ischemic liver was observed under a fluorescence microscope using JC1, as previously described [14]. L02 cells that had not been subjected to $\mathrm{H} / \mathrm{R}$ were used as the negative control (Sham group).

2.11. Immunoblotting Assay. Total proteins from liver tissues subjected to reperfusion for $6 \mathrm{~h}$ or H/R-treated L02 hepatocytes were routinely analyzed with immunoblotting. Before sample loading, the BCA Protein Assay Kits were used to assure the homogeneity of protein concentrations. The antibodies were used at the following concentrations: AR (1:1500), cleaved caspase 3 (1:2000), Bcl-2 (1:2000), $\operatorname{Bax}(1: 2000), \beta$-actin $(1: 2000)$, and secondary antibody $(1: 2500)$. The levels of protein expression were determined with Image J software (NIH, Bethesda, MD).
2.12. Statistical Analysis. The results are expressed as mean \pm SEM. Statistical analysis was performed with an unpaired Student's $t$-test using SPSS 18.0 statistical software. A probability level of $P<0.05$ was considered statistically significant. All results were obtained from at least 5 independent experiments.

\section{Results}

3.1. ARI Protected Fatty Livers against I/R-Induced Impairment and Improved Hepatocyte's Fate. To confirm the homogeneity of steatosis in the mouse livers after HFD feeding, their TG contents were analyzed. As shown in Figure 1(a), the TG levels did not differ in the mouse livers among the Sham, Ctrl, and ARI groups. Morphologically, extensive intrahepatic lipid droplets could be observed both in the H\&E-stained sections and SEM photography (Figure 1(b)).

As direct causes for liver dysfunction following $I / R$, hepatocellular necrosis, apoptosis, and inflammation were analyzed. As shown in Figures 1(b) and 1(c), ARI markedly reduced I/R-mediated hepatic necrosis and inflammatory cell infiltration both in morphological observations and in the quantitative Suzuki scores using H\&E-stained sections. Analogous results were also observed in flow cytometry deployed to quantitate the proportions of apoptotic and necrotic hepatocytes (Figures $1(\mathrm{~d})$ and 1(e)). Furthermore, the ultrastructural assessment indicated that ARI significantly rehabilitated the I/R-induced histological disruptions (Figure 1(b)).

The transaminases ALT and AST are generated within hepatocytes and drastic elevations always denote cellular membranous leakage or hepatocyte disruption caused by hepatic inflammation and/or necrosis. In the present study, I/R-insult dramatically increased serum transaminase levels, whereas the ARI administration significantly palliated these changes (Figures $1(\mathrm{f})$ and $1(\mathrm{~g})$ ).

It is well known that the proper proportions of $\mathrm{Bcl}-2$ family members, especially the antiapoptotic protein $\mathrm{Bcl}$ 2 and the proapoptotic protein Bax, are critical for the maintenance of mitochondrial function and the modulation of the caspase-dependent apoptotic pathway. Meanwhile, caspase 3 is widely accepted as an "executor" for cell apoptosis when it was matured to cleaved form. In this study, ARI markedly enhanced $\mathrm{Bcl}-2$ and the $\mathrm{Bcl}-2 / \mathrm{Bax}$ ratio while it suppressed the activation of caspase 3 at the protein level, although there was no evident change in the Bax protein levels (Figures 2(a)-2(c)).

3.2. ARI Reversed the I/R-Mediated Imbalances in $N A D(P)(H)$ and Redox Status. NADPH is an indispensable coenzyme in the generation of GSH, and the latter acts as the major intracellular ROS-scavenger and may in turn inhibit the formation of MDA, a production of membrane lipid-peroxidation. Therefore, the hepatocellular contents of $\mathrm{NAD}(\mathrm{P})(\mathrm{H}), \mathrm{GSH}$, GSSG, and MDA as well as the proportion of ROS-positive hepatocytes were measured. After ARI treatment, the I/Rinduced decreases in the cytosolic content of NADPH and GSH as well as cytoplasmic NAD were significantly attenuated as compared with the control group, while cytoplasmic 


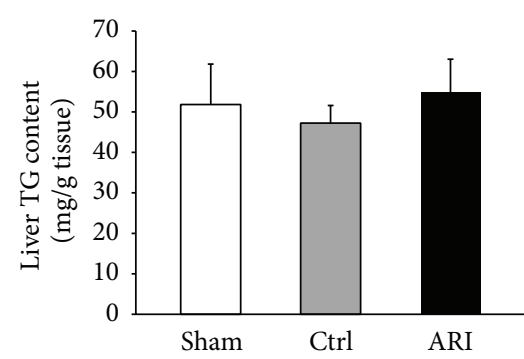

(a)

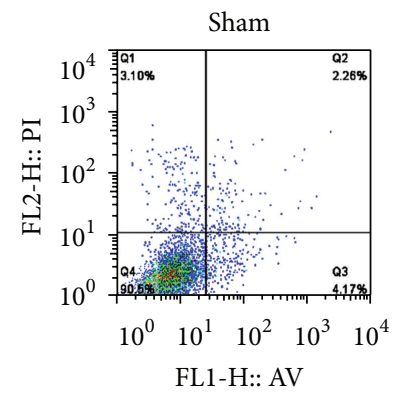

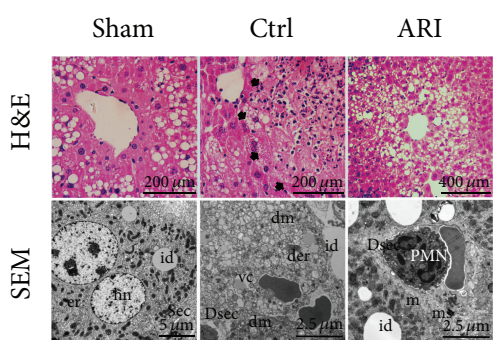

(b)

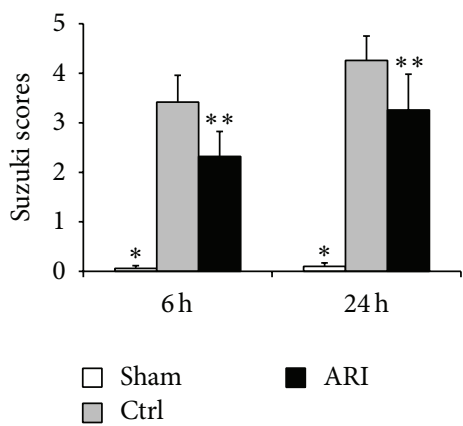

(c)

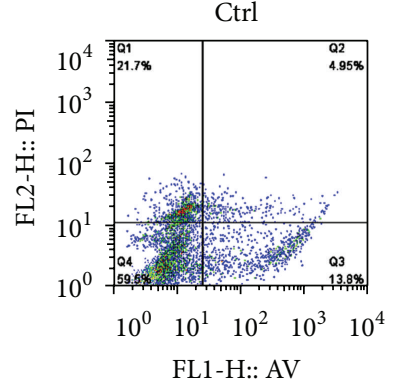

(d)

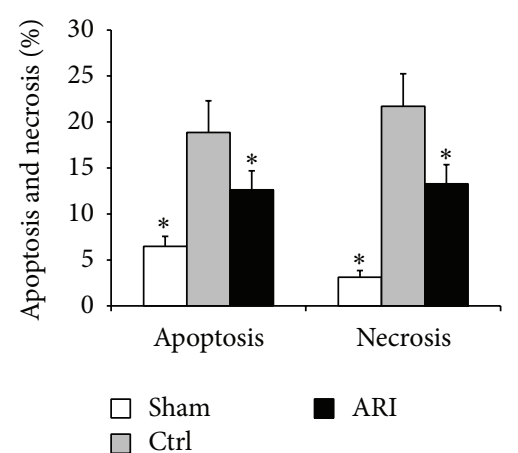

(e)

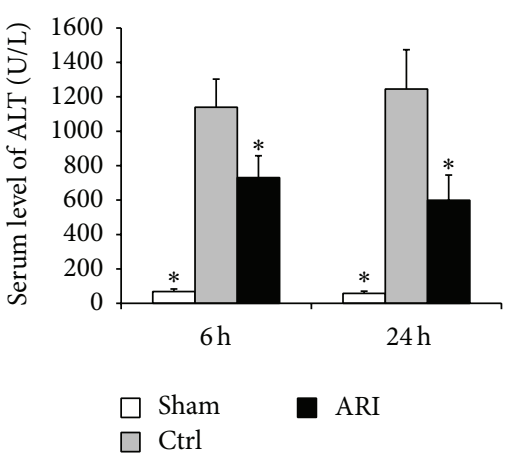

(f)
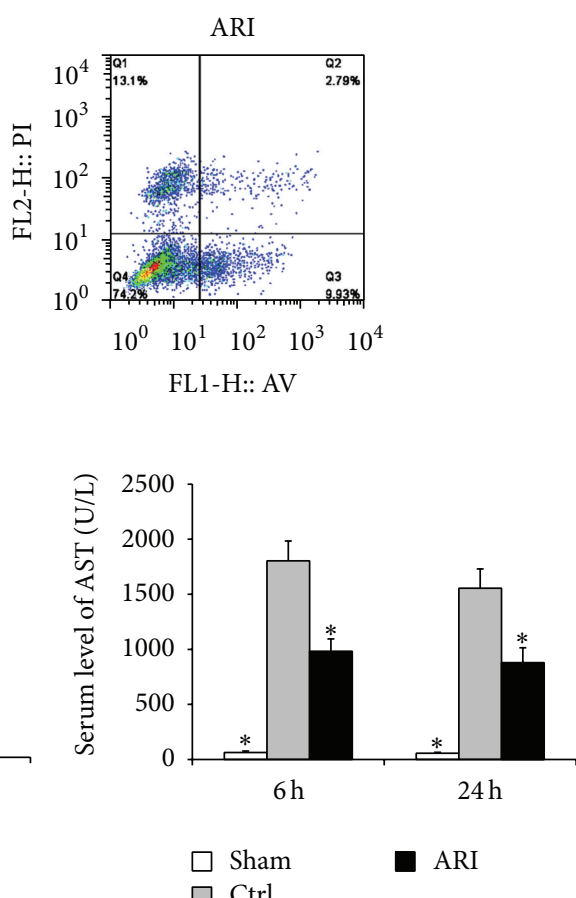

(g)

FIgURE 1: ARI normalized fatty mouse liver function and histology. (a) The hepatic TG levels in Sham, Ctrl, and ARI groups showed no differences after HFD treatment. (b) Both in H\&E staining and SEM, ARI group demonstrated less hepatic necrosis, infiltration of inflammatory cells (black arrow), and ultrastructural disturbances relative to the Ctrl group, whereas Sham group showed an almost intact histological architecture; additionally, intrahepatic lipid droplets are clearly visible. (c) In Suzuki score representing the extent of hepatic necrosis and inflammation, Ctrl group was notably higher than Sham group, and this increase was markedly mitigated by ARI administration. (d)-(e) In the flow cytometry, the Ctrl group showed markedly higher proportions of apoptotic and necrotic hepatocytes than Sham group, and ARI significantly reverted these trends. (f)-(g) I/R insult dramatically elevated serum transaminases (ALT and AST) level, and ARI clearly reversed these changes (for each condition, data are expressed as mean \pm SEM and analyzed by unpaired Student's $t$-test; $n=5$, ${ }^{*} P<0.01$;

$\left.{ }^{* *} P<0.05\right)$. ld, lipid droplet; hn, hepatocyte nucleus; bc, bile canaliculus; ms, microvillus; sec (dsec), (damaged) sinusoidal endothelial cell; m (dm), (damaged) mitochondria; pmn, polymorphonucleocyte; rbc, red blood cell; der, dilated endoplasmic reticulum; ly, lysosome; vc, vacuole.

NADH and cytosolic NADP and MDA contents presented the opposite trends (Figures $2(\mathrm{~d})-2(\mathrm{~g})$ ). On the other hand, remarkable increases could be observed in the NAD/NADH, NADPH/NADP, and GSH/GSSG rates after ARI administration (Figures $2(\mathrm{~h})-2(\mathrm{j})$ ), while the hepatocellular ROSpositive rate presented contrary variation (Figures $2(\mathrm{k})$ and 2(l)).
3.3. AR Overexpression Worsened L02 Hepatocytes' Apoptosis and Elevated Intracellular MDA Level. After steatosis induction, L02 cell from pAR, Ctrl, and GFP groups showed significantly high TG level as compared with Lean group, although there was no marked variance among $\mathrm{pAR}, \mathrm{Ctrl}$, and GFP groups (Figure 3(a)). On FCM using TUNEL staining, AR overexpression evidently deteriorated the H/R-induced 


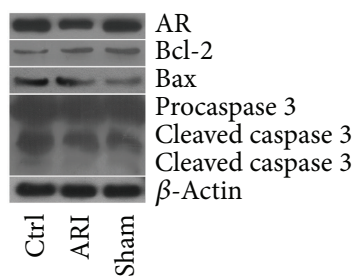

(a)

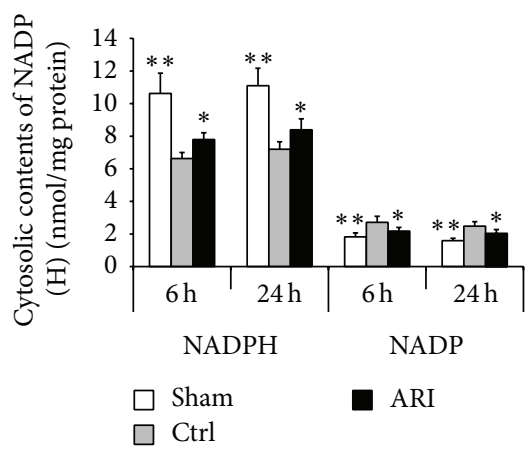

(d)

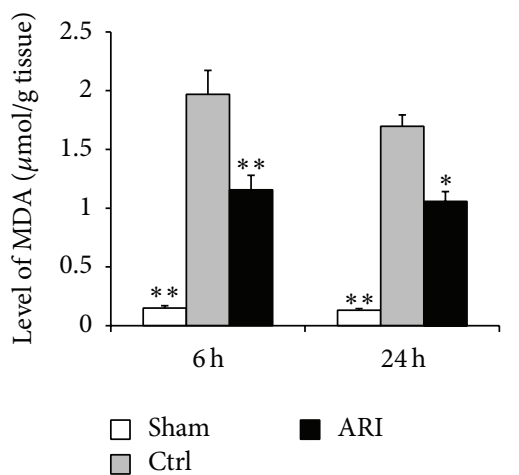

$(\mathrm{g})$

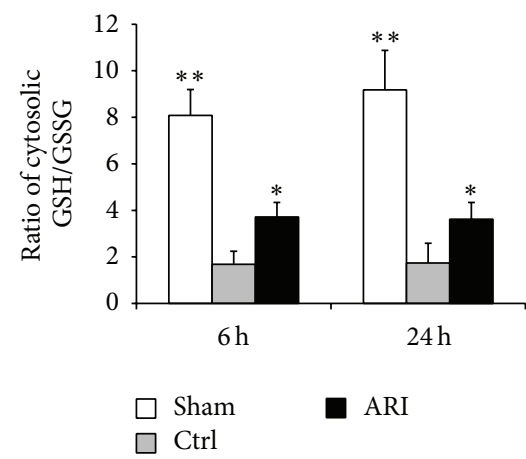

(j)

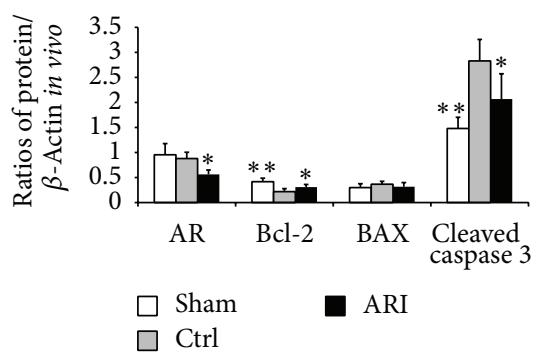

(b)

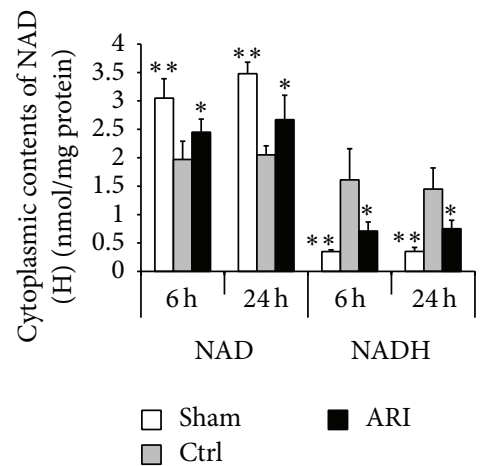

(e)

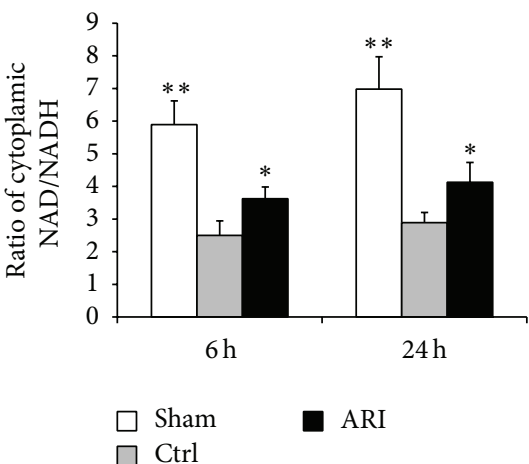

(h)

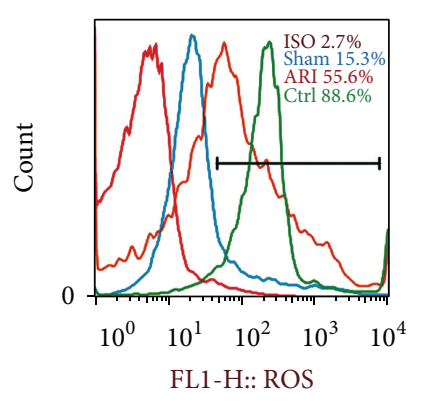

(k)

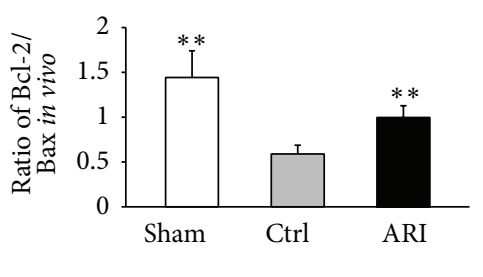

(c)

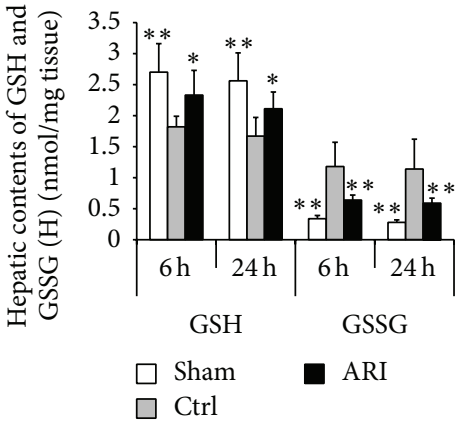

(f)

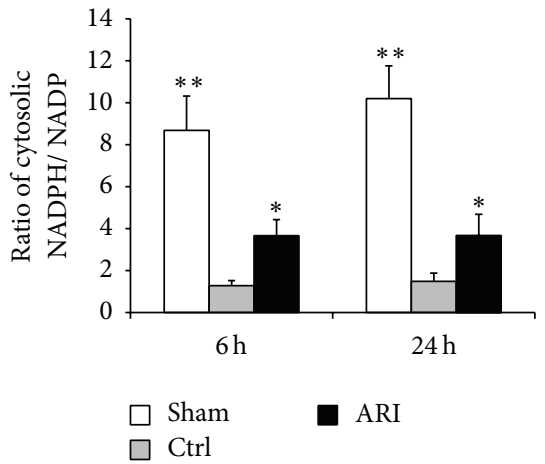

(i)

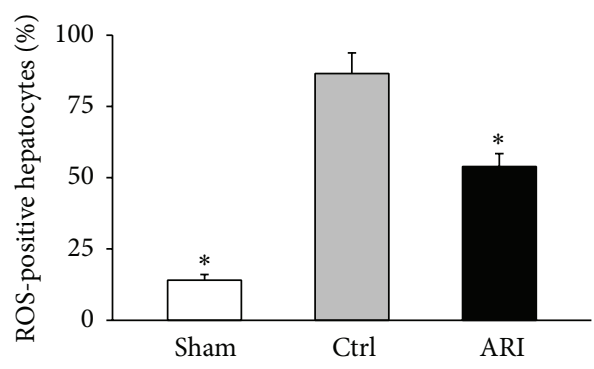

(l)

FIGURE 2: ARI inhibited the caspase-3-dependent apoptosis and reversed the hepatic $\operatorname{NAD}(\mathrm{P})(\mathrm{H})$ contents and redox status imbalance while it lowered ROS content. (a) Representative immunostained photograph. (b)-(c) At the protein level, ARI markedly enhanced Bcl-2 and the $\mathrm{Bcl}-2 / \mathrm{Bax}$ ratio while it inhibited cleaved caspase 3 as compared with the Ctrl group, although there was no marked effect on Bax. (d) $-(\mathrm{g})$ After ARI treatment, the I/R-induced decreases in cytoplasmic NAD and cytosolic NADPH and GSH were significantly attenuated, while cytoplasmic NADH and cytosolic NADP and MDA presented the opposite trends. (h)-(j) Remarkable increases could also be observed in the rates of NAD/NADH, NADPH/NADP, and GSH/GSSG after ARI administration. (k)-(l) In flow cytometry used to detect the proportion of ROS-positive hepatocyte, Ctrl group was significantly higher than Sham group, whereas ARI administration markedly attenuated this variation (for each condition, data are expressed as mean \pm SEM and analyzed by unpaired Student's $t$-test; $n=5,{ }^{*} P<0.05$; ${ }^{* *} P<0.01$ ). 


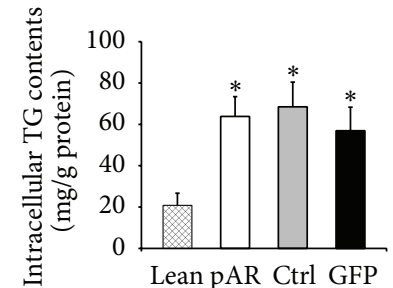

(a)

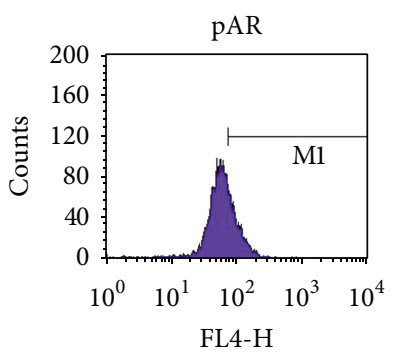

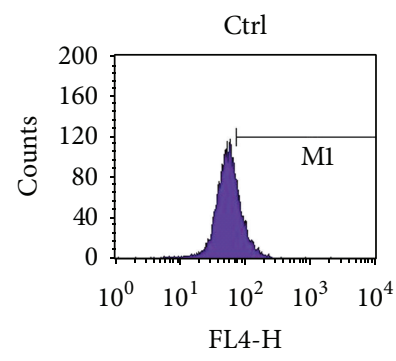

(b)

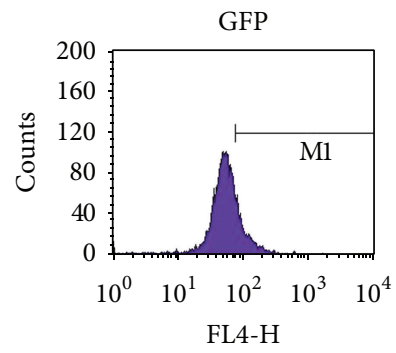

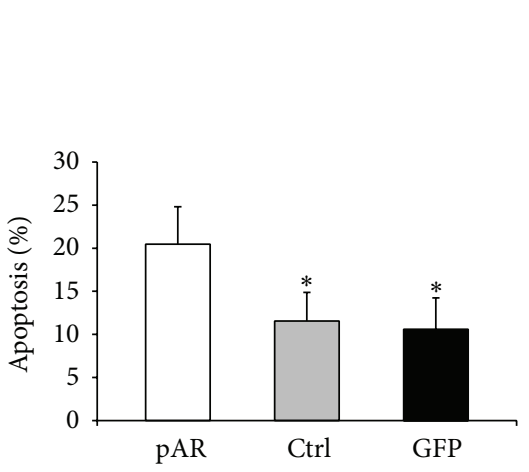

(c)

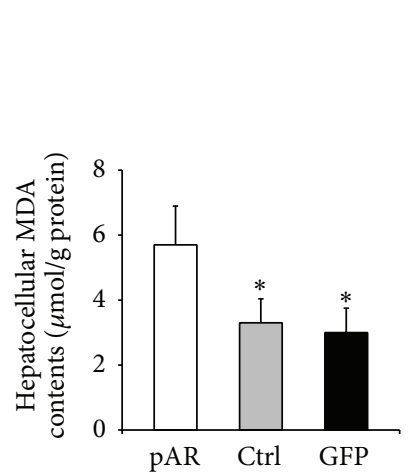

(d)

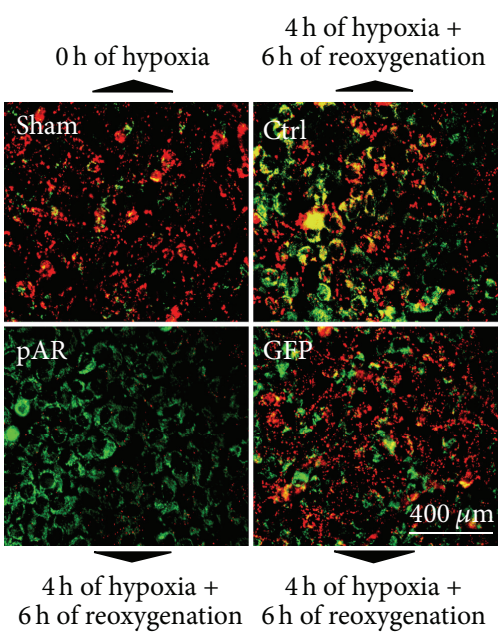

(e)

FIGURE 3: AR-overexpression worsened L02 hepatocytes' apoptosis, elevated intracellular MDA level, and disrupted hepatocytes' mitochondrial membrane potential. (a) After steatosis induction, L02 cell from pAR, Ctrl, and GFP group showed significantly high TG level as compared with Lean group, although there was no marked variance among pAR, Ctrl, and GFP groups. (b)-(c) On FCM using TUNEL staining, AR overexpression evidently deteriorated the H/R-induced apoptosis as compared with Ctrl and GFP group. (d) Meanwhile, pAR transfection also notably elevated the MDA contents when compared to Ctrl and GFP group. (e) In fluorescence using JC-1 staining to detect $\Delta \Psi \mathrm{m}$, the levels of Ctrl and GFP groups were approximately identical, which were clearly lower and higher than those of Sham (Redfluorescent and cytoplasmic dominance) and pAR group (Green-fluorescent and cytomembrane dominance), respectively (for each condition, data are expressed as mean \pm SEM and analyzed by unpaired Student's $t$-test; $n=5,{ }^{*} P<0.01$ ).

apoptosis as compared with Ctrl and GFP groups (Figures 3(b) and 3(c)). Meanwhile, pAR transfection also notably elevated the MDA contents in H/R-treated L02 cell when compared to Ctrl and GFP groups (Figure 3(d)).

\subsection{AR Overexpression Disrupted the Mitochondrial Mem-} brane Potential. After H/R treatment and JC-1 incubation, Ctrl and GFP groups presented with similar $\Delta \Psi \mathrm{m}$ under the fluorescence microscope, being markedly lower than Sham (Red-fluorescent and cytoplasmic dominance) and higher than pAR (Green-fluorescent and cytomembrane dominance) groups (Figure 3(e)).

\subsection{AR Overexpression Activated the I/R-Mediated Caspase-} Dependent Apoptotic Pathway. As shown in Figures 4(a)4(c), the Ctrl group and GFP group showed nearly identical $\mathrm{Bcl}-2$ levels and $\mathrm{Bcl}-2 / \mathrm{Bax}$ ratios, which were both higher than those of the pAR group. In contrast, protein Bax and cleaved caspase 3 were significantly higher in the pAR group.

\section{Discussion}

In this study, two opposite models including in vivo AR inhibition and in vitro AR overexpression were deployed to investigate the role of polyol pathway in ischemic fatty liver. Our results demonstrated that flux through polyol pathway may render steatosis liver greater vulnerability to I/R events, which mainly showed as decreased liver function, disrupted histological architecture, and declined hepatocytes' fates. To be a potential therapeutic target, the role of AR in ischemic steatotic liver should be sufficiently understood and validated.

4.1. Improved NADPH/NADP Ratio and Redox Status. $\mathrm{NADPH}$ is an indispensable cofactor in the reducing two important antioxidant agents, GSH and thioredoxin, from their oxidized forms. As a result, NADPH acts as an indirect scavenger of ROS in the body [15], and also plays an important role in counteracting the inactivation of the antioxidant enzyme catalase. Therefore, it is reasonable to regard NADPH as an ultimate reducing equivalent and a vital 


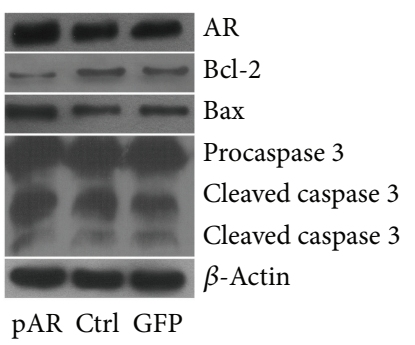

(a)

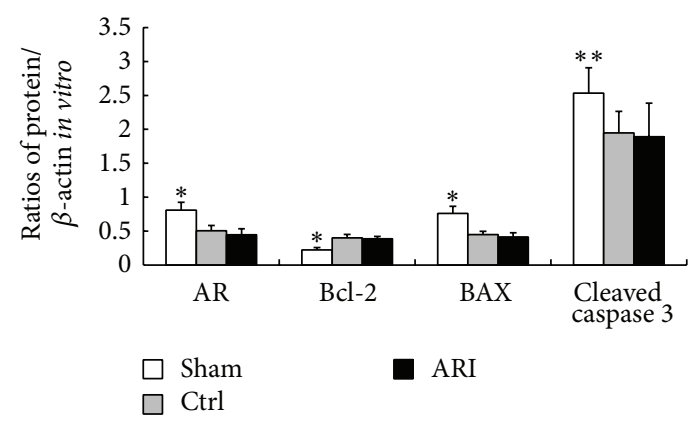

(b)

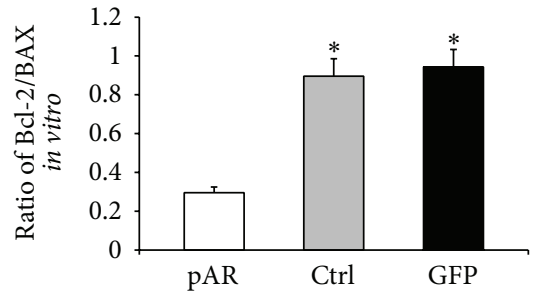

(c)

FIgURE 4: AR-overexpression promoted the activation of caspase 3 in steatosis hepatocytes after H/R. (a)-(c) In western blot, Bcl-2 and Bcl2/Bax ratio were markedly suppressed by AR overexpressing; whereas Bax and cleaved caspase 3 were enhanced (for each condition, data are expressed as mean \pm SEM and analyzed by unpaired Student's $t$-test; $n=5,{ }^{*} P<0.01 ;{ }^{* *} P<0.05$ ).

constituent of the mammalian antioxidant defenses [16, 17]. In the present study, the flux through the polyol pathway dramatically reduced the intracellular contents of NADPH and GSH, which in turn worsened the I/R-induced ROS formation, cellular organellar insult (e.g., membrane lipid peroxidation) and mitochondrial dysfunction. Paradoxically, NADPH also contributes to the generation of superoxide under the catalytic effect of NADPH oxidases (NOXs) [1719]. For this reason, NOXs are generally recognized as the second main source of ROS, only behind the mitochondria. Notably, this phenomenon is almost based on the study of NOXs, but not NADPH $[17,19]$. To the best of our knowledge, NADPH itself remains beneficial during I/R because of its robust reducing properties.

4.2. Elevated NAD/NADH Ratio. During hypoxia, low level of ATP could occur in the liver to sustain the hepatocytes through anaerobic glycolysis mediated by cofactor NAD $[20,21]$. It is plausible that any cause of NAD deficit might exacerbate I/R-induced injury because of the reduced glycolysis. This notion has been supported by several studies, in which NAD depletion following niacin deficiency or poly (ADP-ribose) polymerase overactivation aggravated tissue $\mathrm{I} / \mathrm{R}$ injury, whereas the subsequent repletion of NAD or niacin markedly reversed this phenomenon $[4,22,23]$. The ameliorative effects of the restored NAD/NADH ratio have also been associated with the suppression of the HIF1 $\alpha$ TfR-Tf pathway and the enhanced activation of SIRT1 [5, $14,24,25]$. Until now, little attention has been given to the $\mathrm{NAD} / \mathrm{NADH}$ ratio during hepatic I/R, especially in the fatty liver. Limited to the scale of this study, we did not explore all the courses discussed above, but the thread of NAD began to loom. The flux through the polyol pathway worsened I/R-induced NAD depletion in the fatty liver, which may impair NAD-dependent glycolysis and aggravate energetic failure. Additionally, fatty livers intrinsically have a lower NAD/NADH ratio than lean livers, which might account in part for their increased vulnerability to I/R-insult [26].

4.3. Cross-Talk between Redox Imbalance, Mitochondrial Impairment, and Hepatocyte Fate. Mitochondrion acts as the fundamental generator of intracellular energy and is also involved in multiple hepatocellular function regulations such as redox homeostasis and cell fate [27]. On the other hand, hepatocyte necrosis and apoptosis represent two major direct causes of liver dysfunction following severe I/R impairment and represent different extremes on a continuum of cell death. They share the common triggers of mitochondrial impairment and resultant energy failure [28]. During this cascade, excessive ROS and lipid peroxidation, together with an imbalance in pro- and antiapoptosis proteins (e.g., $\mathrm{Bax}$ and $\mathrm{Bcl}-2$, respectively), promote the mitochondrial permeability transition $[27,29]$. These changes may, in turn, initiate and interact with the following mitochondrial events: respiratory chain uncoupling, the release of cytochrome $c$, the downstream activation of caspase 3 , and so forth [27]. In the present study, the increased $\mathrm{Bax} / \mathrm{Bcl}-2$ ratio which resulted from AR overexpression clearly lowered the mitochondrial membrane potential and contributed to the overactivation of the caspase pathway. However, ARI treatment significantly alleviated the I/R-induced increase of ROS, $\mathrm{Bax} / \mathrm{Bcl}-2$ ratio, and activation of caspase 3, which could eventually improve the liver function. Interestingly, besides its bioactivity in converting glucose to sorbitol, the polyol pathway can also detoxify the noxious aldehydes generated by lipid peroxidation [30]. Therefore, it seemed reasonable that the polyol pathway may protect tissues from I/R-injury by downregulating the intracellular aldehyde content. This notion has been supported by I/R model in which ARI increased the cytosolic aldehyde content and exacerbated tissue ischemic injury [30]. Presumably, these discrepancies may be attributed to the differences in tissue resources, inhibition schemes, the duration of ischemia or reperfusion, and so forth. Besides the polyol pathway, there are many other enzymes responsible for the detoxification of aldehydes, after all $[31,32]$.

Until now, various protocols have been developed to modulate the metabolic processes and/or antioxidant properties of the fatty liver during $I / R$. As the mitochondrion acts as both major victim and the generator of oxidative stress, pharmacological agents aiming at keeping the hepatic energetic balance and mitochondrial function may represent 
a novel strategy for protecting the fatty liver from severe I/Rinduced impairment.

\section{Conclusion}

In conclusion, we used two opposite models, in vivo $\mathrm{AR}$ inhibition and in vitro AR overexpression, to investigate the effects of AR on the fatty liver after I/R. Our study demonstrated that the flux through the polyol pathway renders the fatty liver greater vulnerability to $I / R$ in the forms of decreased liver function and deteriorated hepatocytes fates, which could be evidently rehabilitated by ARI pretreatment. This study demonstrated that strategies that inhibit polyol pathway might provide a novel adjunctive approach to protect the steatotic liver from I/R-insult.

\section{Conflict of Interests}

The authors declare that there is no conflict of interests regarding the publication of this paper.

\section{Acknowledgment}

This work was fully financed by the National Natural Science Foundation of China (no. 81170415). Changhe Zhang and Changjun Huang are co-first authors of this paper.

\section{References}

[1] N. I. Nativ, T. J. Maguire, G. Yarmush et al., "An alternative approach to enable steatotic liver transplantation," The American Journal of Transplantation, vol. 12, no. 12, pp. 3176-3183, 2012.

[2] P. Dutkowski, A. Schlegel, K. Slankamenac et al., "The use of fatty liver grafts in modern allocation systems: risk assessment by the balance of risk (BAR) score," Annals of Surgery, vol. 256, no. 5, pp. 861-868, 2012.

[3] S. Yagihashi, H. Mizukami, S. Ogasawara et al., "The role of the polyol pathway in acute kidney injury caused by hindlimb ischaemia in mice," Journal of Pathology, vol. 220, no. 5, pp. 530541, 2010.

[4] Y. C. Hwang, M. Kaneko, S. Bakr et al., "Central role for aldose reductase pathway in myocardial ischemic injury," The FASEB Journal, vol. 18, no. 11, pp. 1192-1199, 2004.

[5] W. H. Tang, S. Wu, T. M. Wong, S. K. Chung, and S. S. M. Chung, "Polyol pathway mediates iron-induced oxidative injury in ischemic-reperfused rat heart," Free Radical Biology and Medicine, vol. 45, no. 5, pp. 602-610, 2008.

[6] J. Trebicka, I. Racz, S. V. Siegmund et al., "Role of cannabinoid receptors in alcoholic hepatic injury: steatosis and fibrogenesis are increased in CB2 receptor-deficient mice and decreased in CB1 receptor knockouts," Liver International, vol. 31, no. 6, pp. 860-870, 2011.

[7] J. Martin, P. Romanque, O. Maurhofer et al., "Ablation of the tumor suppressor histidine triad nucleotide binding protein 1 is protective against hepatic ischemia/reperfusion injury," Hepatology, vol. 53, no. 1, pp. 243-252, 2011.

[8] M. N. Berry and D. S. Friend, "High-yield preparation of isolated rat liver parenchymal cells: a biochemical and fine structural study," Journal of Cell Biology, vol. 43, no. 3, pp. 506$520,1969$.
[9] J.-Z. Huang, Y.-Z. Chen, M. Su et al., "Dl-3-n-Butylphthalide prevents oxidative damage and reduces mitochondrial dysfunction in an $\mathrm{MPP}^{+}$-induced cellular model of Parkinson's disease," Neuroscience Letters, vol. 475, no. 2, pp. 89-94, 2010.

[10] K. Gong and W. Li, "Shikonin, a Chinese plant-derived naphthoquinone, induces apoptosis in hepatocellular carcinoma cells through reactive oxygen species: a potential new treatment for hepatocellular carcinoma," Free Radical Biology and Medicine, vol. 51, no. 12, pp. 2259-2271, 2011.

[11] S. Suzuki, L. H. Toledo-Pereyra, F. J. Rodriguez, and D. Cejalvo, "Neutrophil infiltration as an important factor in liver ischemia and reperfusion injury: modulating effects of FK506 and cyclosporine," Transplantation, vol. 55, no. 6, pp. 1265-1272, 1993.

[12] M. J. Gómez-Lechón, M. T. Donato, A. Martínez-Romero et al., "A human hepatocellular in vitro model to investigate steatosis," Chemico-Biological Interactions, vol. 165, no. 2, pp. 106-116, 2007.

[13] T. Abe, M. Unno, H. Takeuchi et al., "A new free radical scavenger, edaravone, ameliorates oxidative liver damage due to ischemia-reperfusion in vitro and in vivo," Journal of Gastrointestinal Surgery, vol. 8, no. 5, pp. 604-615, 2004.

[14] A. R. Barbu, G. Akusjärvi, and N. Welsh, "Adenoviral-induced islet cell cytotoxicity is not counteracted by $\mathrm{Bcl}-2$ overexpression," Molecular Medicine, vol. 8, no. 11, pp. 733-741, 2002.

[15] W. Ying, "NAD ${ }^{+} / \mathrm{NADH}$ and $\mathrm{NADP}^{+} / \mathrm{NADPH}$ in cellular functions and cell death: regulation and biological consequences," Antioxidants and Redox Signaling, vol. 10, no. 2, pp. 179-206, 2008.

[16] H. N. Kirkman and G. F. Gaetani, "Mammalian catalase: a venerable enzyme with new mysteries," Trends in Biochemical Sciences, vol. 32, no. 1, pp. 44-50, 2007.

[17] C. A. Benavente and E. L. Jacobson, "Niacin restriction upregulates NADPH oxidase and reactive oxygen species (ROS) in human keratinocytes," Free Radical Biology and Medicine, vol. 44, no. 4, pp. 527-537, 2008.

[18] K. T. Moe, N. O. Yin, T. M. Naylynn et al., "Nox2 and Nox4 mediate tumour necrosis factor- $\alpha$-induced ventricular remodelling in mice," Journal of Cellular and Molecular Medicine, vol. 15, no. 12, pp. 2601-2613, 2011.

[19] C. Piccoli, G. Quarato, A. D’Aprile et al., "Native LDL-induced oxidative stress in human proximal tubular cells: multiple players involved," Journal of Cellular and Molecular Medicine, vol. 15, no. 2, pp. 375-395, 2011.

[20] P.-W. So and B. J. Fuller, "Enhanced energy metabolism during cold hypoxic organ preservation: studies on rat liver after pyruvate supplementation," Cryobiology, vol. 46, no. 3, pp. 295300, 2003.

[21] J. T. Brosnan, H. A. Krebs, and D. H. Williamson, "Effects of ischaemia on metabolite concentrations in rat liver," Biochemical Journal, vol. 117, no. 1, pp. 91-96, 1970.

[22] C. Siegel and L. D. Mccullough, "NAD" depletion or PAR polymer formation: which plays the role of executioner in ischaemic cell death?" Acta Physiologica, vol. 203, no. 1, pp. 225234, 2011.

[23] W. Ying, P. Garnier, and R. A. Swanson, "NAD ${ }^{+}$repletion prevents PARP-1-induced glycolytic blockade and cell death in cultured mouse astrocytes," Biochemical and Biophysical Research Communications, vol. 308, no. 4, pp. 809-813, 2003.

[24] S. Y. Jang, H. T. Kang, and E. S. Hwang, "Nicotinamide-induced mitophagy: event mediated by high $\mathrm{NAD}^{+} / \mathrm{NADH}$ ratio and SIRT1 protein activation," Journal of Biological Chemistry, vol. 287, no. 23, pp. 19304-19314, 2012. 
[25] Q. Yu, T. Wang, X. Zhou et al., "WldS reduces paraquat-induced cytotoxicity via SIRT1 in non-neuronal cells by attenuating the depletion of NAD," PLoS ONE, vol. 6, no. 7, Article ID e21770, 2011.

[26] H.-J. Kim, J. H. Kim, S. Noh et al., "Metabolomic analysis of livers and serum from high-fat diet induced obese mice," Journal of Proteome Research, vol. 10, no. 2, pp. 722-731, 2011.

[27] S. W. Ryter, P. K. Hong, A. Hoetzel et al., "Mechanisms of cell death in oxidative stress," Antioxidants and Redox Signaling, vol. 9, no. 1, pp. 49-89, 2007.

[28] H. Jaeschke, "Reactive oxygen and mechanisms of inflammatory liver injury: present concepts," Journal of Gastroenterology and Hepatology, vol. 26, supplement 1, pp. 173-179, 2011.

[29] G. Loor, J. Kondapalli, H. Iwase et al., "Mitochondrial oxidant stress triggers cell death in simulated ischemia-reperfusion," Biochimica et Biophysica Acta: Molecular Cell Research, vol. 1813, no. 7, pp. 1382-1394, 2011.

[30] K. Wetzelberger, S. P. Baba, M. Thirunavukkarasu et al., "Postischemic deactivation of cardiac aldose reductase: role of glutathione S-transferase $\mathrm{P}$ and glutaredoxin in regeneration of reduced thiols from sulfenic acids," Journal of Biological Chemistry, vol. 285, no. 34, pp. 26135-26148, 2010.

[31] J. M. Guo, A. J. Liu, P. Zang et al., "ALDH2 protects against stroke by clearing 4-HNE," Cell Research, vol. 23, no. 7, pp. 915930, 2013.

[32] J. J. Chen and B. P. Yu, "Detoxification of reactive aldehydes in mitochondria: effects of age and dietary restriction," Aging, vol. 8, no. 5, pp. 334-340, 1996. 


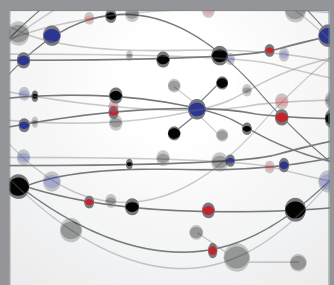

The Scientific World Journal
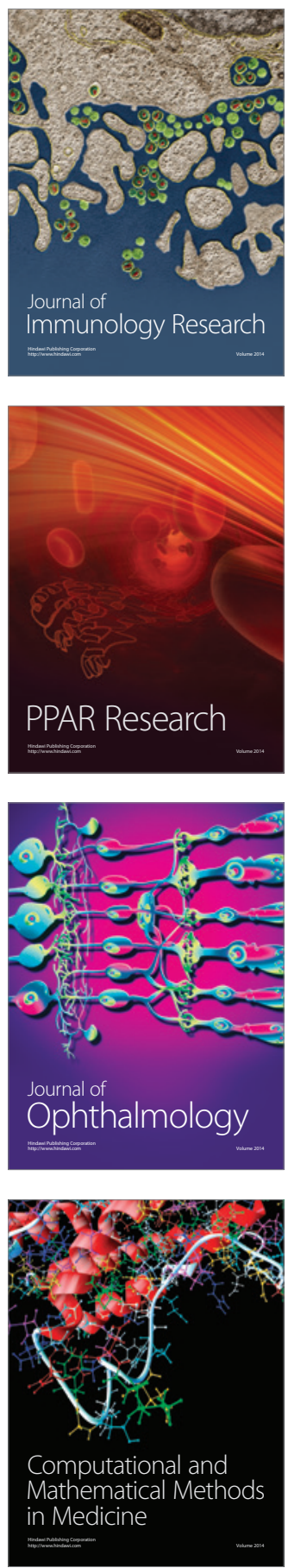

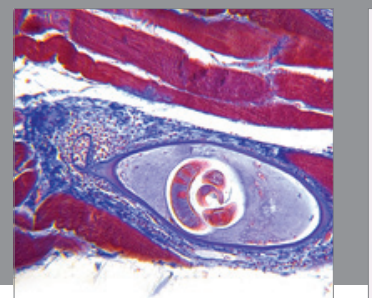

Gastroenterology

Research and Practice
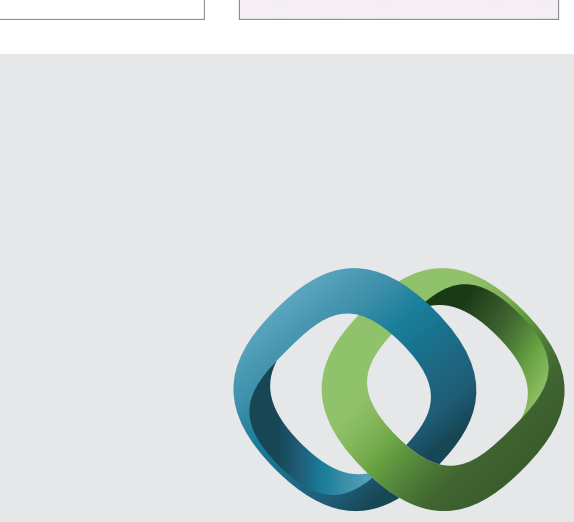

\section{Hindawi}

Submit your manuscripts at

http://www.hindawi.com
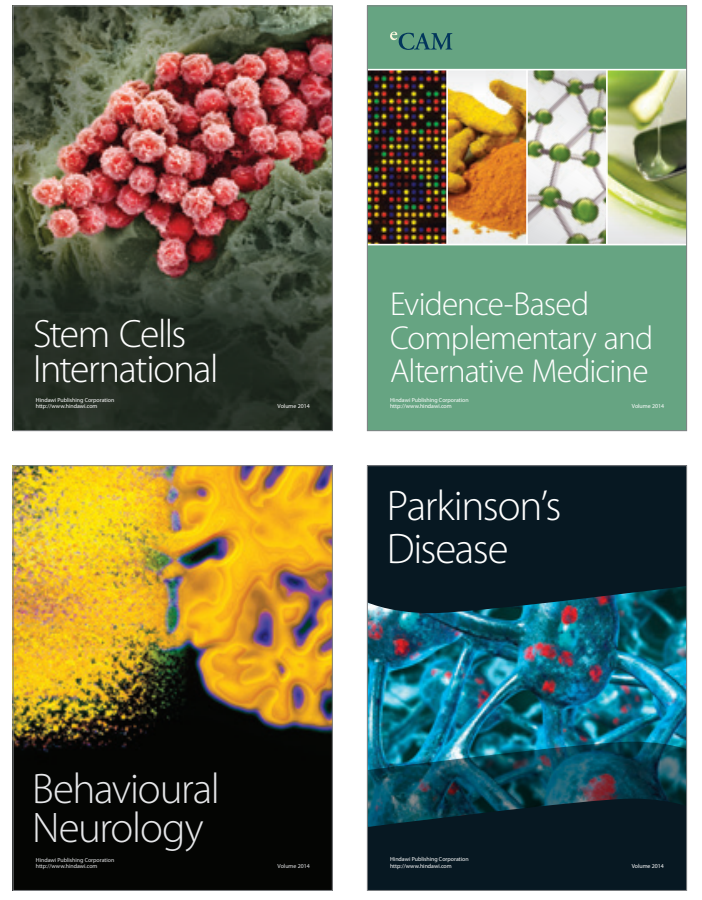
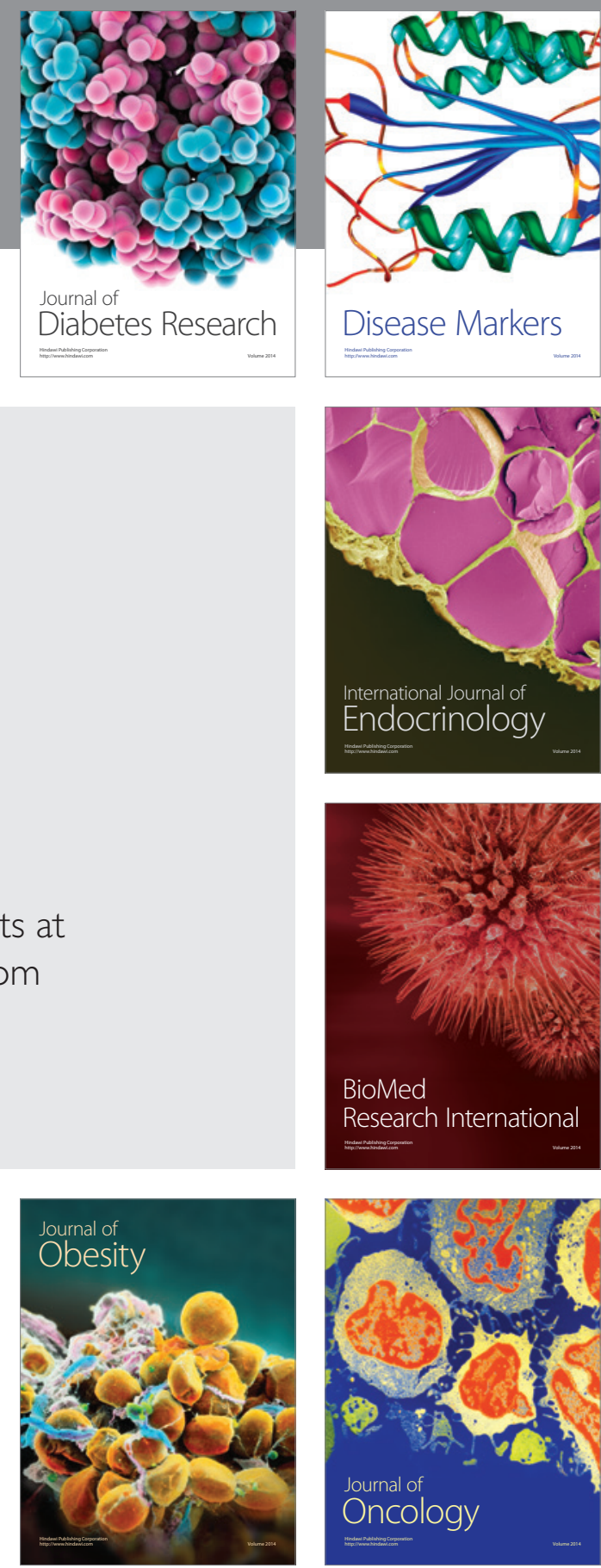

Disease Markers
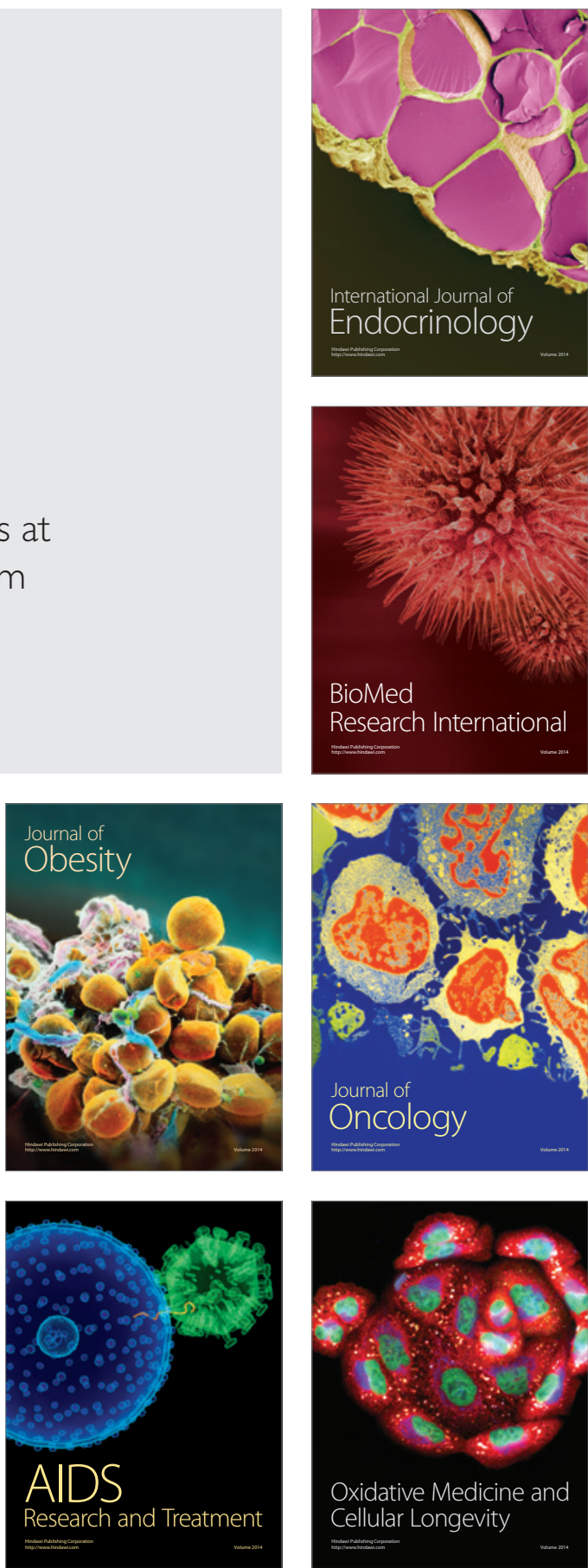